\title{
Metastatic mandibular ameloblastoma of the lung ten years after primary resection: A rare case report
}

\author{
Konstantinos Kapriniotis $^{1}$, Angelina Bania ${ }^{1}$, Savvas Lampridis ${ }^{1}$, Georgios Geropoulos ${ }^{1}$, Sofoklis Mitsos ${ }^{1}$, \\ Fleur Monk ${ }^{2}$, David A. Moore ${ }^{2}$, Nikolaos Panagiotopoulos ${ }^{1}$ \\ ${ }^{1}$ Department of Thoracic Surgery; ${ }^{2}$ Department of Histopathology, University College London Hospitals NHS \\ Foundation Trust, London, UK
}

\begin{abstract}
Ameloblastoma is a rare odontogenic neoplasm of the jaw. It usually behaves as a benign, slow growing tumour of the oral cavity with a high recurrence rate, especially when it is inadequately resected. A small proportion of ameloblastomas metastasize to distant organs, with lungs representing the most common site of metastatic spread. In this report, we present the case of a middle-
\end{abstract}

Correspondence: Konstantinos Kapriniotis, University College Hospital at Westmoreland Street, 16-18 Westmoreland Street, London, W1G 8PH, UK.

Tel. +44.7463640559

E-mail: konstantinos.kapriniotis@nhs.net

Key words: metastatic ameloblastoma; jaw tumour; lung metastasis.

Authors' Contributions: KK, GG, NP, conception and design of study; AB, GG, administrative support; SM, FM, DM, acquisition of study materials or patients; KK, AB, data analysis and interpretation. All authors, manuscript drafting and reviewing. All the authors have read and approved the final version of the manuscript and agreed to be accountable for all aspects of the work.

Conflict of interest: The authors declare no conflict of interest.

Funding: This study did not receive any funding.

Patient consent for publication: Written informed consent was obtained from the patient for publication of this Case Report and accompanying images. A copy of the written consent is available for review by the Editor-in Chief of this journal on request.

Conference presentation: This case report has been submitted as an eposter at the $13^{\text {th }}$ Panhellenic Congress of the Hellenic Society of Thoracic and Cardiovascular Surgeons, 5-7/11/2020, Athens, Greece.

Received for publication: 17 October 2020

Accepted for publication: 31 January 2021.

${ }^{\circ}$ Copyright: the Author(s), 2021

Licensee PAGEPress, Italy

Monaldi Archives for Chest Disease 2021; 91:1643

doi: 10.4081/monaldi.2021.1643

This article is distributed under the terms of the Creative Commons Attribution Noncommercial License (by-nc 4.0) which permits any noncommercial use, distribution, and reproduction in any medium, provided the original author(s) and source are credited. aged man with two pulmonary nodules and a history of mandibular ameloblastoma excised 10 years prior to this radiological finding. Following resection and histopathological analysis of the lung lesion, a diagnosis of metastatic ameloblastoma was confirmed. No local recurrence of the primary tumour was identified. At 1-year follow-up, the patient had no evidence of local or metastatic disease.

\section{Introduction}

Ameloblastoma is a rare neoplasm arising from the epithelium of the dental lamina. It represents only $1 \%$ of all oral neoplasms and approximately $10 \%$ of odontogenic tumours. The incidence of ameloblastoma is estimated at 1-2 cases per million people every year and peaks during the $3^{\text {rd }}$ and $4^{\text {th }}$ decade of life. The tumour presents more frequently in Asian and African populations compared to Caucasians, without showing gender predisposition [1,2]. Regarding the pathogenesis of ameloblastoma, tumour cells are considered to originate from cell rests of the enamel organ, residual epithelium of the tooth germ or epithelium of odontogenic cysts. Ameloblastomas most frequently occur spontaneously but can also be triggered by trauma or inflammation [3]. From a clinical perspective, they usually behave as benign, slow growing but locally aggressive oral tumours, with a high rate of local recurrence if inadequately resected. As low as $2 \%$ of ameloblastomas present with distant metastasis. Metastatic ameloblastomas may present with benign histological features and indolent clinical behaviour of both the primary and metastatic site. Contrariwise, amenoblastic carcinomas present with malignant histological characteristics and more aggressive clinical course, regardless of the presence or not of distant metastasis at the time of diagnosis $[4,5]$. Herein, we report the case of a middle-aged man with two pulmonary nodules and a history of mandibular ameloblastoma excised 10 years prior to this radiological finding.

\section{Case Report}

A 53-year-old male patient of Asian origin was referred to our clinic due to the presence of two lung lesions suspicious for malignancy. The pulmonary nodules had been identified after he underwent chest radiograph and computed tomography (CT) for the investigation of persisting productive cough. No additional respiratory or constitutional symptoms were reported. His past medical history included mandibular amenoblastoma treated with left mandibulectomy and reconstruction with left fibular graft 10 years 
prior to this presentation. The patient was regularly followed up by the maxillofacial surgical team and there were no indications of local tumour recurrence. He did not receive any regular medications and he was a smoker with a history of 40 pack-years. Clinical examination revealed normal findings from the respiratory system and no signs of mandibular tumour recurrence, peripheral lymphadenopathy or hepatosplenomegaly.

A chest CT scan revealed two lesions in the left lung: a calcified nodule $12 \mathrm{~mm}$ in diameter in the upper lobe and a nodule $10 \mathrm{~mm}$ in diameter in the lower lobe. A subsequent positron emission tomography-computed tomography (PET-CT) scan from vertex to proximal thighs demonstrated mild uptake of fluorodeoxyglucose (FDG) in lower lobe nodule, with no FDG activity in the other nodule or other sites (Figure 1). Fine needle aspiration under CT guidance of the lower lobe nodule was performed but the histopathological analysis was inconclusive. Blood tests were within normal range. Pulmonary function tests showed forced expiratory volume in 1 second and diffusing capacity of the lung for carbon monoxide more than $90 \%$ of the predicted values.

The patient underwent wedge resection of both lung lesions, which were sent for frozen section. The upper lobe lesion showed no evidence of dysplasia or malignancy, whereas the lower lobe nodule presented features consistent with non-small cell lung carcinoma. Based on the reported findings, left lower completion lobectomy and systematic mediastinal lymph node dissection were undertaken. The patient had an uncomplicated recovery and was discharged on the third postoperative day.

Histopathological examination of the excised nodule after tissue fixation revealed anastomosing islands of proliferating odontogenic epithelium set amongst hyalinised stroma with abundant squamoid epithelium (Figures 2 and 3). Genetic analysis of the tumour detected a driver mutation in the BRAF gene (Val600Glu). Previous studies have demonstrated that the majority of ameloblastomas harbour this driver mutation in BRAF. Given the above findings and the patient's past medical history, a diagnosis of metastatic ameloblastoma was confirmed. The surgical margins were negative for microscopic residual tumour and the excised lymph nodes were clear of metastatic disease. Given the complete excision of the metastasis and the absence of local recurrence at the site of the primary tumour, decision was made for active surveillance. At 1year follow-up, the patient had no complications or evidence of tumour recurrence on clinical examination and CT imaging.

\section{Discussion}

Several cases have been published in the English literature under the terms 'malignant ameloblastoma', 'metastasizing ameloblastoma' or 'atypical ameloblastoma'. However, it remains unclear how many of these cases truly represented diagnoses of metastatic ameloblastoma according to the diagnostic criteria of the World Health Organisation, since metastatic ameloblastoma and ameloblastic carcinoma were initially not considered different pathological entities [5]. Indeed, Van Dam et al. who reviewed 98 cases under the above terms, from the first reported case in 1923 until 2010 , concluded that only 27 of these represented valid reports of metastasizing (malignant) ameloblastoma, $81 \%$ of which originated in the mandible. Lungs were the initial site of metastasis in $78 \%$ of the cases, of which $71 \%$ were bilateral. Interestingly, the primary mandibular tumour recurred on average four times before metastasis [6]. This comes in contrast with our case, in which no local recurrence occurred prior to identification of metastasis.

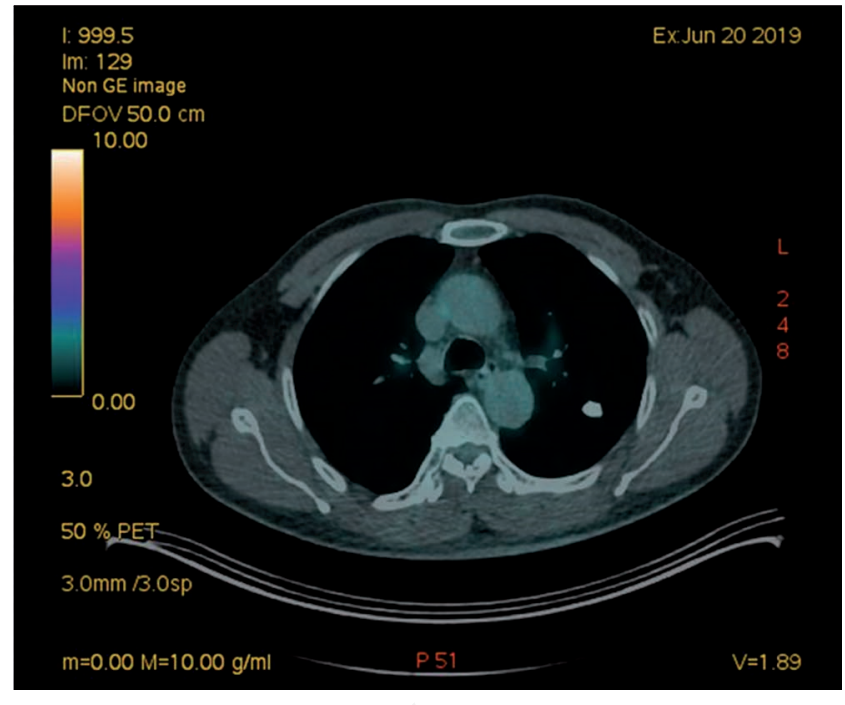

Figure 1. PET CT scan, mildly FDG avid left lower lobe lesion.

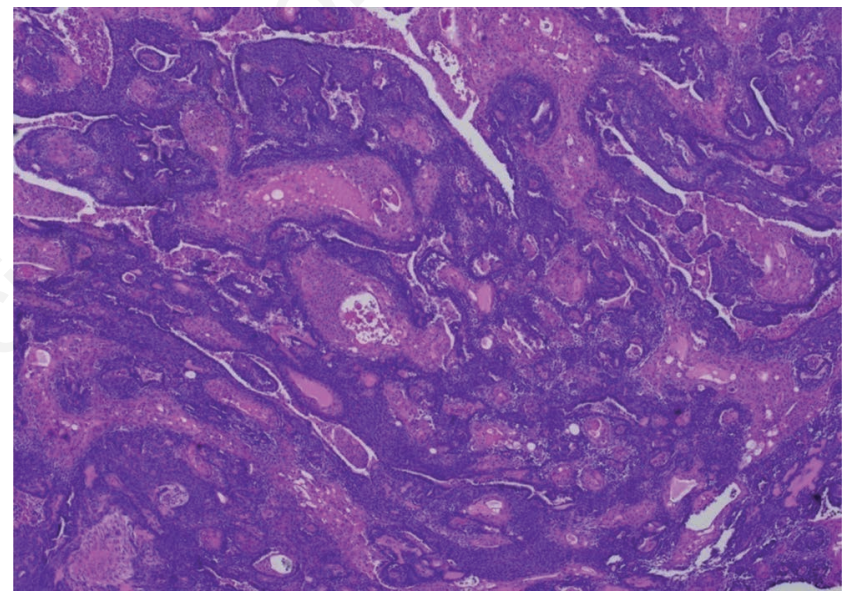

Figure 2. Low power view showing anastomosing islands of proliferating odontogenic epithelium set amongst hyalinised stroma with abundant squamoid epithelium.

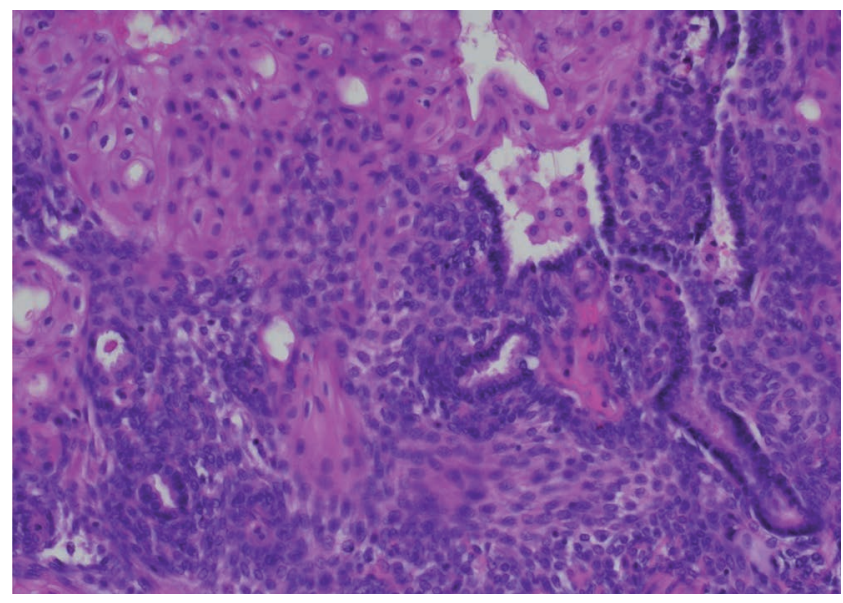

Figure 3. High power view showing basophilic columnar ameloblasts displaying characteristic peripheral palisading and reverse polarized nuclei with inconspicuous mitotic figures. 
The lungs are considered to be the most common site of metastasis for ameloblastomas, representing $75 \%-88 \%$ of all metastatic cases, followed by regional lymph nodes of the neck [6-8]. Other sites of distant disease reported in the literature include the vertebrae, brain, kidneys, skin and heart [9-11]. The time interval between diagnosis of the primary tumour and the metastasis is typically long, but it can vary between a few months and 45 years. Van Dam et al. reported an average time from diagnosis of primary tumour to metastasis of 18 years, whereas Laughlin et al. reported a mean disease $\square$ free interval between diagnosis of tumour and appearance of metastasis of 9 years in 43 patients [6,7].

Regarding the pathophysiology of the metastatic course, several theories have been proposed. Haematogenous spread is considered to be the most likely route of metastasis. This theory is strongly supported by the commonly reported bilateral involvement of the lungs $[6,12]$. Some authors favour the lymphatic route of metastasis considering the relatively shorter disease-free interval in lymph node metastasis compared to lung metastasis and the simultaneous lymph node and lung metastases in some cases [7]. Finally, aspiration has been also proposed as possible metastatic pathway, mostly supported by the endobronchial localization of some lung metastases and the more frequent localization of metastasis in the right lung [13]. No factors have been identified as predictors of metastatic spread. However, it appears that the size of the primary tumour, incomplete surgical resection, non-surgical or conservative surgical management and the number of local recurrences may affect the possibility of late metastatic disease $[6,14]$.

Due to the limited experience with this rare condition, there is no unanimously accepted follow-up protocol to screen for late metastatic spread. Many authors suggest long-term follow-up, in the form of annual clinical examination of the primary site, respiratory tract, neck lymph nodes and muscosceletal system and annual radiographic evaluation of the primary site and chest. In case of abnormal findings in the primary work-up, further investigation with computed tomography of the chest is imperative [15].

There is still controversy regarding the optimal management of ameloblastoma with lung metastasis. Typically, management of operable lesions includes pulmonary metastasectomy, with wedge resection or segmentectomy preferred to lobectomy, in an attempt to preserve pulmonary function. Effective surgical management of the metastatic site has offered a prolonged, disease-free survival in several cases reported in the literature up to date $[6,14]$.

Radiation therapy and chemotherapy, alone or in combination, are used when the metastatic disease is non-operable or for palliation of symptoms related to the disease. Several chemotherapeutic agents have been used for the management of ameloblastoma, including adriamycin, bleomycin, vinblastine, cisplatin, carboplatin, paclitaxel, 5-fluouracil and methotrexate. The outcomes of cytotoxic chemotherapy for metastatic ameloblastoma are still controversial, since previous cases suggest a wide range of responses, from chemoresistance to considerable symptom and tumor size improvement $[16,17]$.

Recently, biological targeted therapies, including BRAF gene inhibitors, have shown promising results in some cases of metastasizing ameloblastoma. BRAF mutations have been detected in a high proportion of ameloblastoma cases, typically exceeding $50 \%$ according to a recent systematic review [18]. Early case reports present an impressive short-term response of both primary and metastatic sites to BRAF inhibitors, succeeding considerable symptomatic relief and tumour size reduction [19]. However, the follow-up period in these reports is typically very short. Therefore, larger studies with longer follow-up periods are needed to evaluate
BRAF inhibitors effect on survival rates and establish their role in the management of metastatic ameloblastoma.

In total, there are no large comparative studies evaluating the effect of the different management options on disease course and overall survival rates. Most of the available evidence comes from a heterogeneous group of case reports and small retrospective studies. Of note is that metastatic disease in the lung usually has a benign clinical behaviour and some reports suggest long-term survival without any management of the metastatic site [20]. Therefore, some authors have favoured active surveillance of metastatic lung lesions, especially in asymptomatic patients not suitable for surgical resection. It is of paramount importance these patients to be followed with regular imaging in order to detect early disease progression.

Prognosis of metastatic disease is rather favourable compared to other cancers with pulmonary metastases, considering that metastases of ameloblastoma usually follow the indolent course of the primary site. In the series reported by Van Dam et al., 12 of 25 had an average survival time of approximately 3 years after diagnosis of metastasis. The remaining 13 patients were still alive at the time their reports were made and had survived an average of 10 years since the diagnosis of metastasis [6].

\section{Conclusions}

Although relatively uncommon, metastatic ameloblastoma of the lung should be included in the differential diagnosis of suspicious lung nodules in all patients with previously resected mandibular ameloblastoma. Interestingly, metastatic disease typically occurs many years after primary site resection, even without local recurrence of the primary tumour, and presents with indolent clinical behaviour and favourable prognosis. Surgical resection, either conservative or wide, is considered to be the treatment of choice in most of these cases. Cytotoxic chemotherapy, radiotherapy or BRAF inhibitors may provide clinical benefit for non-operable lesions.

\section{References}

1. Oomens MA, Van der Waal I. Epidemiology of ameloblastomas of the jaws; a report from the Netherlands. Med Oral Patol Oral Cir Bucal 2014;19:e581-3.

2. MacDonald-Jankowski DS, Yeung R, Lee KM, Li TK. Ameloblastoma in the Hong Kong Chinese. Part 2: systematic review and radiological presentation. Dentomaxillofac Radiol 2004;33:141-51.

3. Nagi R, Sahu S, Rakesh N. Molecular and genetic aspects in the etiopathogenesis of ameloblastoma: An update. J Oral Maxillofac Pathol 2016;20:497-504.

4. Verneuil A, Sapp P, Huang C, Abemayor E. Malignant ameloblastoma: classification, diagnostic, and therapeutic challenges. Am J Otolaryngol 2002;23:44-8.

5. Thompson L. World Health Organization classification of tumours: pathology and genetics of head and neck tumours. Ear Nose Throat J 2006;85:74.

6. Van Dam SD, Unni KK, Keller EE. Metastasizing (malignant) ameloblastoma: review of a unique histopathologic entity and report of Mayo Clinic experience. J Oral Maxillofac Surg 2010;68:2962-74. 
7. Laughlin EH. Metastasizing ameloblastoma. Cancer 1989;64: 776-80.

8. Jayaraj G, Sherlin HJ, Ramani P, et al. Metastasizing Ameloblastoma - a perennial pathological enigma? Report of a case and review of literature. J Craniomaxillofac Surg 2014;42: 772-9.

9. White RM, Patterson JW. Distant skin metastases in a longterm survivor of malignant ameloblastoma. J Cutan Pathol 1986;13:383-9.

10. Hayakawa K, Hayashi E, Aoyagi T, et al. Metastatic malignant ameloblastoma of the kidneys. Int J Urol 2004;11:424-6.

11. Nguyen BD. Malignant ameloblastoma with thoracic vertebral metastasis: PET/CT and MR imaging. Clin Nucl Med 2005;60: 450-2.

12. Azumi T, Nakajima T, Takeuchi S, et al. Malignant ameloblastoma with metastasis to the skull: report of case. J Oral Surg 1981;39:690-6

13. Vorzimer J, Perla D. An Instance of adamantinoma of the Jaw with Metastases to the Right Lung. Am J Pathol 1932;8:445-54.

14. Rizzitelli A, Smoll NR, Chae MP, et al. Incidence and overall survival of malignant ameloblastoma. PLoS One 2015;10: e0117789.

15. Berger AJ, Son J, Desai NK. Malignant ameloblastoma: concurrent presentation of primary and distant disease and review of the literature. J Oral Maxillofac Surg 2012;70:2316-26.

16. Grünwald V, Le Blanc S, Karstens JH, et al. Metastatic malignant ameloblastoma responding to chemotherapy with paclitaxel and carboplatin. Ann Oncol 2001;12:1489-91.

17. Lanham RJ. Chemotherapy of metastatic ameloblastoma. A case report and review of the literature. Oncology 1987;44:133-4.

18. González-González R, López-Verdín S, Lavalle-Carrasco J, et al. Current concepts in ameloblastoma-targeted therapies in Braf proto-oncogene serine/threonine kinase V600E mutation: Systematic review. World J Clin Oncol 2020;11:31-42.

19. Broudic-Guibert M, Blay JY, Vazquez L, et al. Persistent response to vemurafenib in metastatic ameloblastoma with BRAF mutation: a case report. J Med Case Rep 2019;13:245.

20. Hasim FW, Poon CC, Smith AC. Prolonged survival with confirmed metastatic pulmonary ameloblastoma. Int $\mathrm{J}$ Oral Maxillofac Surg 2007;36:953-5. 